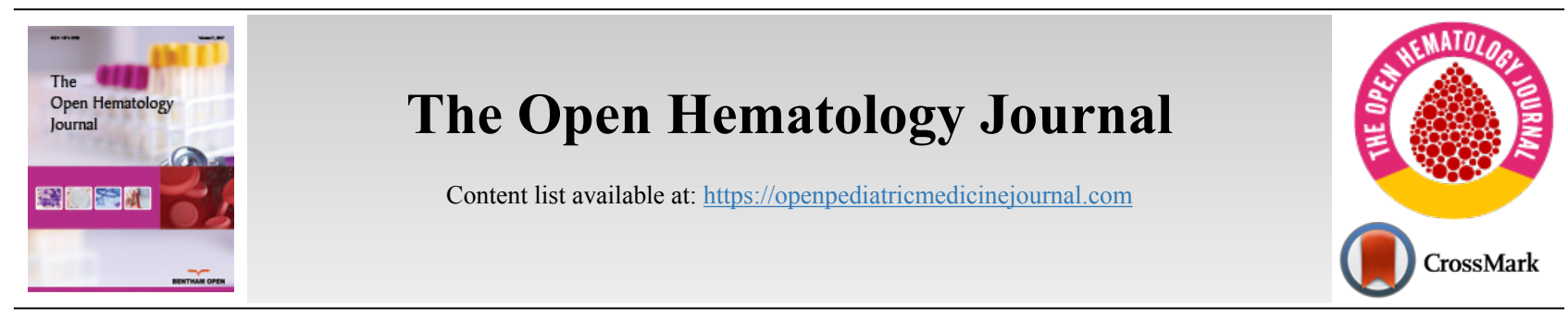

CASE REPORT

\title{
Dengue Fever Complicated By Hemophagocytosis - A Difficult To Manage Case
}

\author{
Saravanan Thangavelu*, Clement Jenil Dhas and Mohammad Zeya Ansari \\ Department of General Medicine PSG Institute of Medical Sciences and Research, Coimbatore, India
}

\begin{abstract}
Dengue fever, which is transmitted by the mosquito Aedes aegypti is a common acute viral febrile illness, affecting 390 million people worldwide every year. It is endemic in several countries. Although the usual presentation is that of a self-limiting illness, its complications are protean. Infection Associated with Hemophagocytic Syndrome (IAHS) is a rare and fatal complication of dengue fever. It should be suspected in a patient with fever beyond seven days, associated with hepatosplenomegaly, hyperferritinemia, worsening cytopenias and development of multiorgan dysfunction(MOD). We report a 19-year-old female, who presented with an acute febrile illness and was diagnosed with primary dengue fever. Despite appropriate supportive therapy, she worsened clinically during the course of hospitalisation. A disproportionately high ferritin level and persistent bicytopenia prompted investigations for hemophagocytic lymphohistiocytosis (HLH). Further evaluation revealed features of HLH, as per the diagnostic criteria laid down by the Histiocyte Society. She was successfully treated with glucocorticoids, etoposide and intravenous immunoglobin and other supportive therapy. She had severe cytopenia and cardiac dysfunction during the course of her illness and its management. This case adds to the limited adult cases of dengue-associated hemophagocytic syndrome and shows its difficulty in management due to associated extra complications.
\end{abstract}

Keywords: Dengue, Cytokine storm, MOD, IAHS, HLH, Hemophagocytosis.

\begin{tabular}{|l|l|l|l}
\hline Article History & Received: October 09, 2018 & Revised: January 06, 2019 & Accepted: February 01, 2019
\end{tabular}

\section{INTRODUCTION}

Dengue fever which is endemic in India and several other countries can sometimes manifest with many atypical features sometimes that may be life-threatening too if not diagnosed and intervened in time [1] HLH is one of them, which is now being detected more readily due to increasing awareness amongst clinicians. It is a status of abnormal immune response caused by a cytokine storm induced by the uncontrolled proliferation and activation of the macrophages in the reticuloendothelial system causing a systemic inflammatory response and multi-organ dysfunction [2]. Reactive HLH is associated with infections, autoimmune disorders or malignancies. HLH has been reported in dengue patients [3]. Its incidence is estimated to be approximately 1.2 cases per $10,000,000$ individuals [4]. Primary HLH occurs due to various genetic abnormalities and often presents during infancy and early childhood. It is associated with an autosomal recessive inheritance pattern. Secondary HLH is associated with infections, malignancy, metabolic and rheumatological conditions. Many infections are known to cause HLH and this is often mistaken for sepsis and multiorgan dysfunction syndrome(MODS). We describe the case of a 19-year-old

\footnotetext{
* Address correspondence to this author at the Department of General Medicine PSG Institute of Medical Sciences and Research, Coimbatore, India; Tel: +914224345160/5166; E-mail: dr_saravanan12@yahoo.co.in
}

female, who had primary dengue fever complicated by hemophagocytosis which was difficult to manage due to different drug and non-drug related complications.

\subsection{Case Report}

A 19-year-old female was admitted with high-grade intermittent fever for 3 days, associated with a headache and myalgia without any symptoms suggestive of a focus of infection. On the day of admission, she had mild pallor, no icterus or edema and her vitals were stable. Systemic examination did not reveal any significant finding. Her white blood cell count was 1800 cells/cu.mm, hemoglobin was $8.7 \mathrm{~g} / \mathrm{dl}$ and platelet count was 60,000 cells/cu.mm. Liver function tests were moderately deranged with elevated transaminase, alkaline phosphatase and Gama glutaryl transferase. With clinical features and preliminary reports, she was diagnosed to have dengue fever and it was confirmed with dengue IgM positivity (by ELISA). She was treated with supportive care, intravenous fluids and antipyretics. Follow up of cultures was also negative for any organisms.

But even after 6 days of illness, she continued to have a fever and general unwellness. There was an increasing trend in serial liver enzymes, with mild prolongation of prothrombin time. However, she had no bleeding manifestation. She was suspected to have hospital acquired new infection and was evaluated for the same. She started on an empirical intravenous 
broad-spectrum antibiotic as per hospital antibiotic policy. Urine, blood and bone marrow culture were sterile. But she continued to have a fever and general unwellness and worsening of liver function and prothrombin time as well. Serology for human immunodeficiency virus, hepatitis-B, C, A, E and scrub typhus infections was negative. Epstein-Bar virus serology was consistent with past infection while IgM antibody for cytomegalovirus was positive. Anti-nuclear antibody profile was also negative.

On the ninth day of illness, she was shifted to the medical intensive care unit in view of desaturation and worsened sensorium. She was intubated and mechanical ventilation was given. Since she had a worsening course of illness even after on appropriate antibiotic therapy, the possibility of HLH was considered. She underwent a panel of investigations for the same, which included serum triglycerides, ferritin, lactate dehydrogenase $(\mathrm{LDH})$, peripheral smear and bone marrow smear and biopsy, repeat cultures and procalcitonin (Trend of laboratory results shown in Fig. (1). She had high levels of serum triglyceride, ferritin, LDH and evidence of hemophagocytosis in the bone marrow examination (as shown in Figs.
(2 and 3). Fig. (4) Peripheral smear showing spherocytes, suggestive of the transfused population.

Apart from supportive care, she was also started on treatment for hemophagocytic lympho- histiocytosis as per HLH 2004 protocol. She continued to have fever and biochemical parameters showed no improvement even after two days of therapy with Dexamethasone and Etoposide. Hence she was also started on Immunoglobulin therapy. Subsequently, fever and other abnormal parameters regressed. She was extubated and shifted out of the intensive care unit. During her stay in the intensive care unit, she had worsening of neutropenia secondary to Inj. Etoposide, for which she was given Inj. Granulocyte colony-stimulating factor (G-CSF). She also developed left ventricular systolic dysfunction probably as a complication of dengue and/or secondary to immunoglobulin therapy which was managed with fluid restriction and diuretic. After stabilization, she was shifted to ward where she underwent a complete rehabilitation programme. At the time of discharge, her biochemical and hematological parameters normalized. During the three months follow up, she maintains good health and blood parameters.

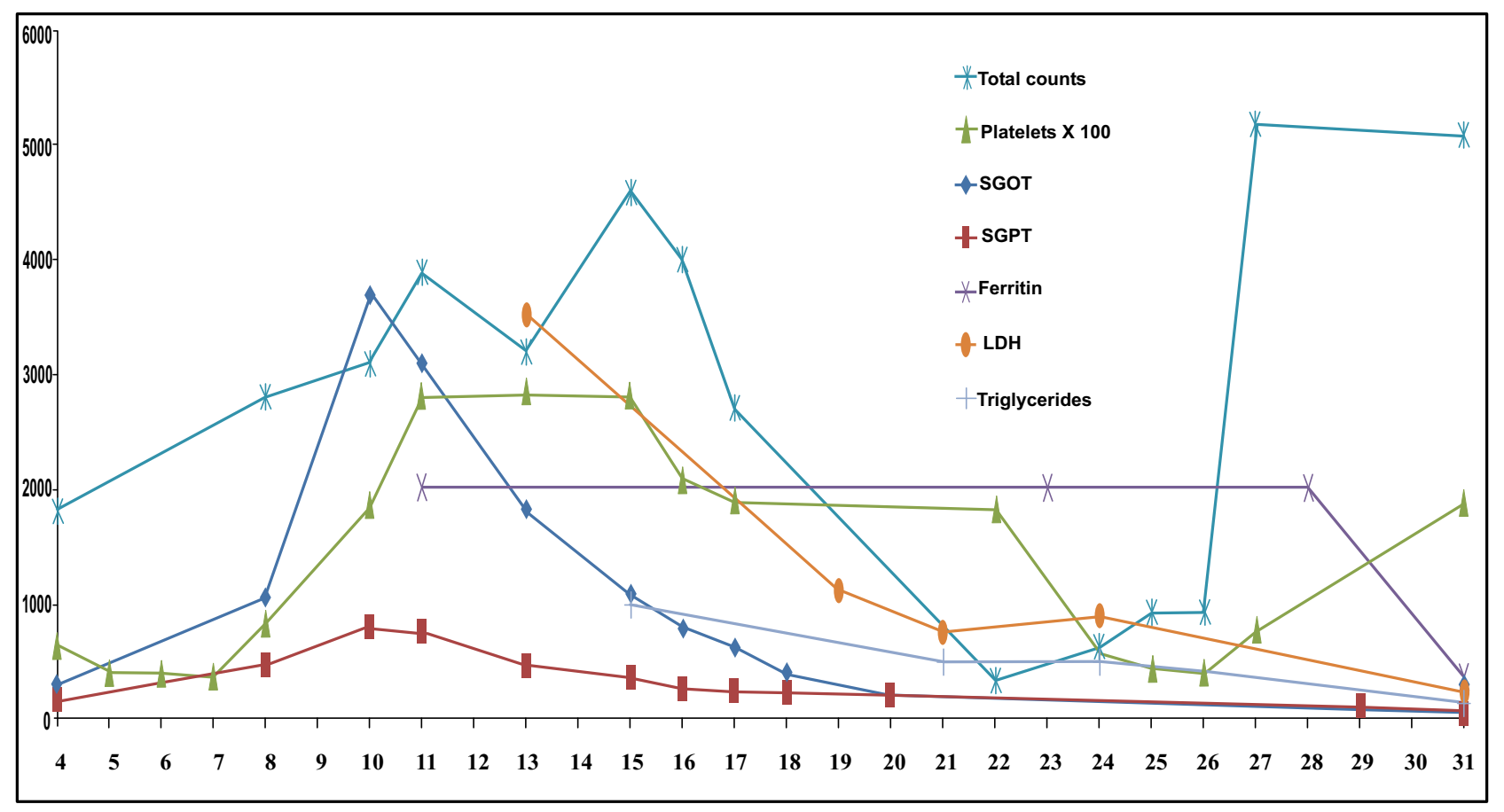

\section{DASOF LLNESS}

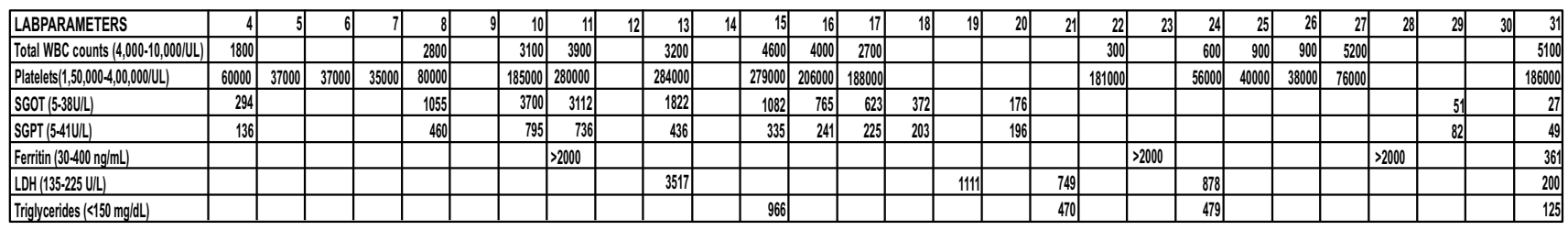

Fig. (1). Showing trend of laboratory results. 


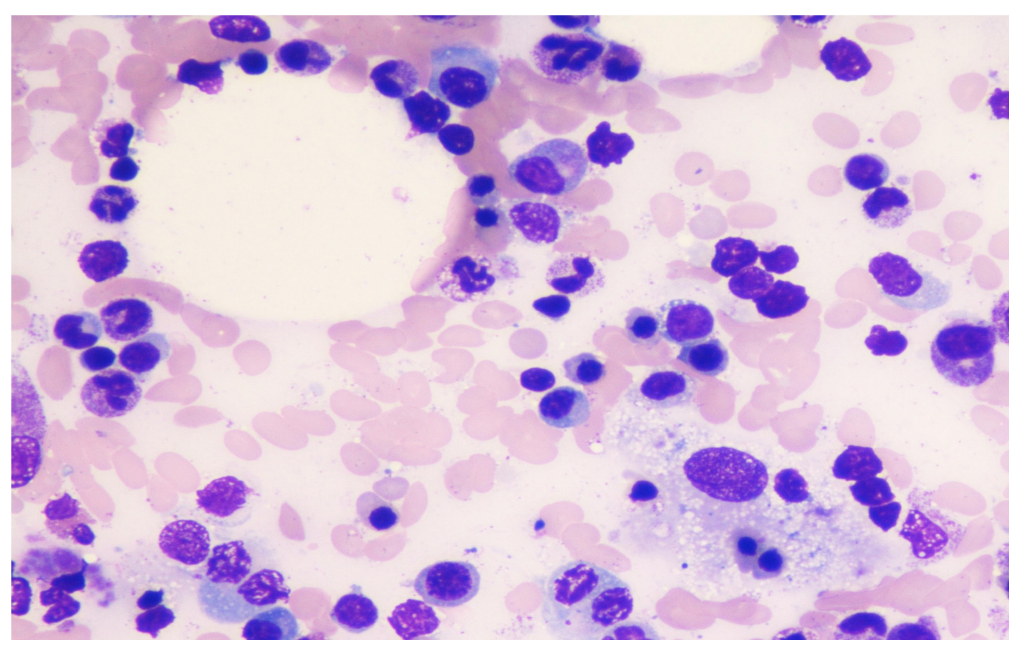

Fig. (2). Bone marrow aspirate showing prominent phagocytosis of blood elements by Histiocytes. (Low Power magnification).

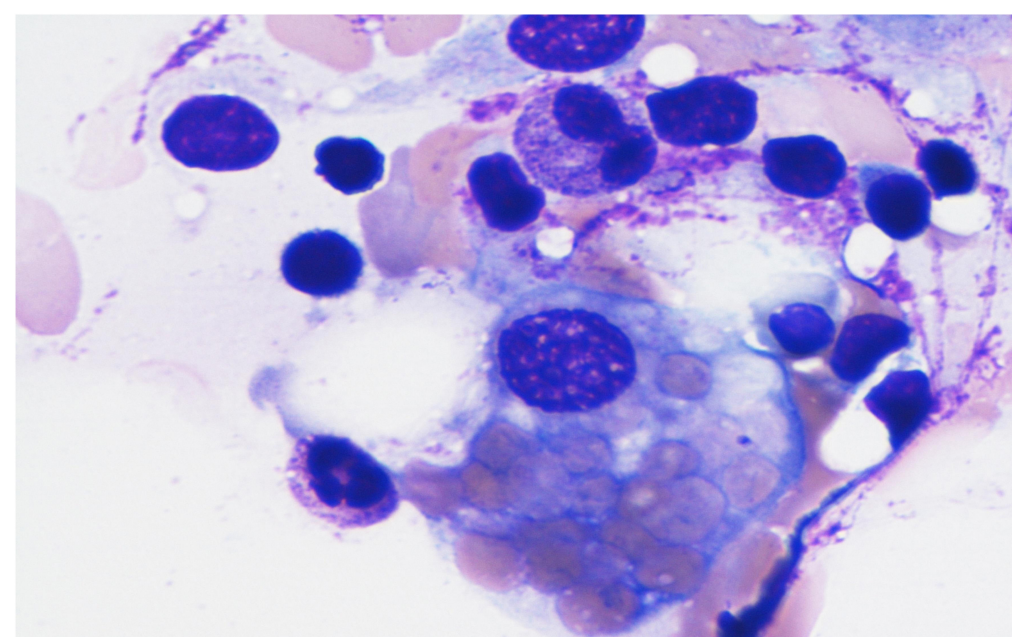

Fig. (3). Bone marrow aspirate showing erythrophagocytosis (High power magnification).

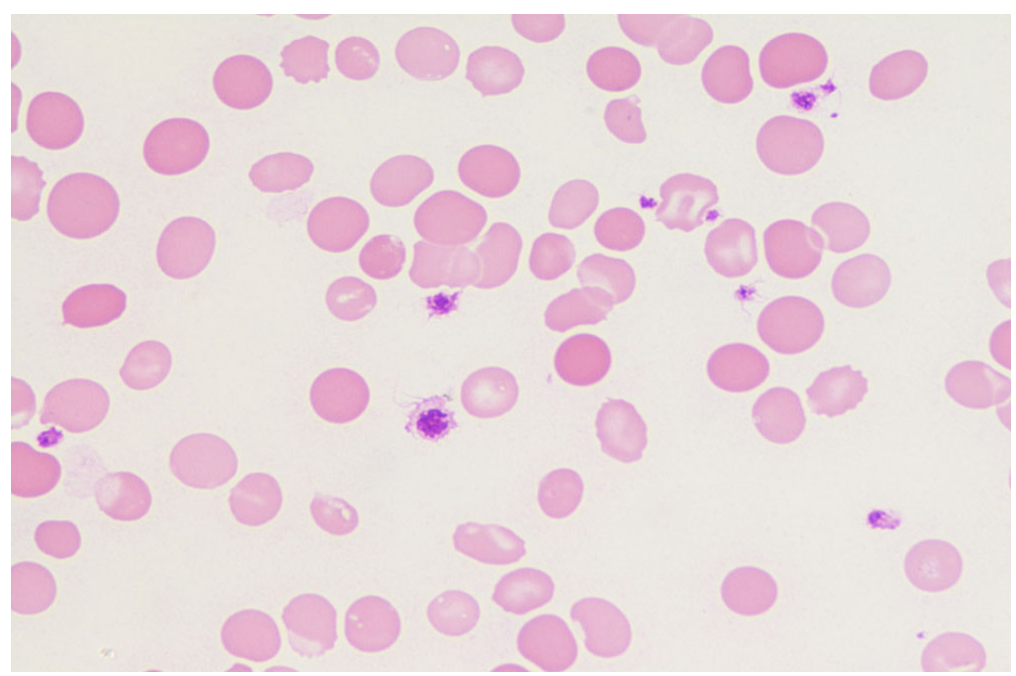

Fig. (4). Peripheral smear with spherocytes suggestive of transfused population (X100). 


\section{RESULTS AND DISCUSSION}

$\mathrm{HLH}$ is a rare and life-threatening disorder with major therapeutic and diagnostic difficulties. It causes tissue destruction due to abnormal immune activation. Clinical features of $\mathrm{HLH}$, which is secondary to unrestrained immune activation are not specific and mimic sepsis with MODS. Although primary HLH is typically seen in the paediatric age group, secondary HLH can occur in adults and children in association with certain triggers which includes solid organ malignancies, infections, rheumatological disorders and inherited or acquired immunodeficiency. Several infections like visceral leishmaniasis [5], tuberculosis, dengue and scrub typhus trigger HLH.

Dengue is an uncommon cause of HLH and has been mentioned in a few case reports [6]. T cells infected with dengue virus produce cytokines like tumor necrosis factor (TNF)- $\alpha$ and interferon (IFN) gamma which possibly contribute to the development of HLH syndrome. HLH is characterized by excessive macrophage activation and cytokine release. This results in unchecked inflammation due to immune dysregulation. Patients with HLH are acutely ill with fever, hepatosplenomegaly, effusions and lymphadenopathy. Laboratory findings include bicytopenia, coagulopathy, liver dysfunction, hyperferritinemia and elevated triglycerides. Bone marrow infiltration by activated macrophages can be demonstrated, and diagnosis is made based on the HLH-2004 protocol proposed by the Histiocyte Society. HLH can be established in the presence of (i) molecular diagnosis consistent with HLH or (ii) the presence of five out of eight criteria, namely, fever, splenomegaly, cytopenias, hypertriglyceridemia and/or hypofibrinogenemia, hemophagocytosis in tissue, hyperferritinemia, increase in CD25/IL-2 receptor and reduced or absent NK cell activity. Our patient met 6 of the 8 criteria.

Glucocorticoids are used as the first line of treatment of HLH. Other agents as per HLH-2004 protocol [2] are cyclosporin, etoposide and intrathecal methotrexate (for patients with central nervous system involvement). The presence of both dengue-associated HLH and multiorgan dysfunction (MOD) in majority of the patients may indicate that they are part of an inflammatory spectrum, which also includes systemic inflammatory response syndrome [7]. Interleukin (IL)-6 and TNF are associated in the pathogenesis of MOD. Nonetheless, treatment for each syndrome is different, as HLH is treated with chemo-immunotherapy whereas supportive therapy is a keystone in MOD.

Dengue fever, caused by a mosquito-borne virus belonging to the Flaviviridae family, is a serious public health problem in the Indian subcontinent. The year 2017 has seen a gross increase in the number of dengue cases in India. The clinical manifestations range from a self-limiting febrile illness to lifethreatening disease with shock and MOD. In a study focusing on 162 adult $\mathrm{HLH}$ patients, $58 \%$ survival was noted [8]. The diagnosis of dengue is based on the detection of virus nonstructural protein 1 , which has greater sensitivity in primary infection $(90 \%)$ when compared with secondary infection (60-80\%). Alternatively, real-time PCR tests are available for virus detection. Serological testing for IgM and IgG antibodies helps in differentiation between primary and secondary infections.
Dengue-associated HLH has been described commonly in children, with few reports in adults [9]. It has been more commonly noted in patients with dengue hemorrhagic fever. But our patient didn't have any bleeding manifestations. In our patient, the diagnosis was established on Day 12 of illness. During the treatment she had cardiac dysfunction probably due to the immunoglobulin administered or dengue itself, hence fluid management was prioritised. She also developed etoposide-induced severe neutropenia which was managed with G-CSF injections. She was successfully treated with etoposide, immunoglobulin and a tapering course of corticosteroids as per the HLH-2004 protocol [10] and showed a good response to the same. At the first month and subsequent monthly follow up, she was well with no features of reactivation. In our patient, the initial improvement was followed by clinical worsening leading to a diagnosis of dengue-associated HLH. This case highlights the need for awareness of causes of worsening dengue, its immediate confirmation and early therapeutic intervention as this may be life-saving. This case also adds to the few adults reported HLH in dengue as there have been approximately 45 cases dengue-related HLH [11].

\section{CONCLUSION}

Usually, dengue fever spontaneously subsides after five days of illness with supportive care alone. If a person continues to be symptomatic even after five to seven days of illness, along with the possibility of secondary bacterial infections there should be a high index of suspicion of its atypical and rare complications. Early diagnosis and intervention may be life-saving.

\section{ETHICS APPROVAL AND CONSENT TO PARTICIPATE}

This study has been approved by the Institutional Human Ethics Committee of PSG Institute of Medical Sciences and Research.

\section{HUMAN AND ANIMAL RIGHTS}

No Animals were used in this research. All human research procedures followed were in accordance with the ethical standards of the committee responsible for human experimentation (institutional and national), and with the Helsinki Declaration of 1975, as revised in 2013.

\section{CONSENT FOR PUBLICATION}

A written informed consent was obtained from all patients when they were enrolled.

\section{CONFLICT OF INTEREST}

The authors declare that there is no conflict of interest, financial or otherwise.

\section{ACKNOWLEDGEMENTS}

Declared none.

\section{REFERENCES}

[1] Dengue Challenges India's Health System [Internet]. [17 October 2015] 
http://www.sciencedirect.com/science/article/pii/S014067361500313X

[2] Henter JI, Horne A, Aricó M, et al. HLH-2004: Diagnostic and therapeutic guidelines for hemophagocytic lymphohistiocytosis. Pediatr Blood Cancer 2007; 48(2): 124-31.

[http://dx.doi.org/10.1002/pbc.21039] [PMID: 16937360]

[3] Ramanathan M, Duraisamy G. Haemophagocytosis in dengue haemorrhagic fever: A case report. Ann Acad Med Singapore 1991; 20(6): 803-4.

[PMID: 1803975]

[4] Ellis EM, Sharp TM, Pérez-Padilla J, et al. Incidence and risk factors for developing dengue-associated hemophagocytic lymphohistiocytosis in puerto rico, 2008 - 2013. PLoS Negl Trop Dis 2016; 10(8): e0004939.

[http://dx.doi.org/10.1371/journal.pntd.0004939] [PMID: 27556807]

[5] Rajagopala S, Dutta U, Chandra KS, Bhatia P, Varma N, Kochhar R. Visceral leishmaniasis associated hemophagocytic lymphohistiocytosis case report and systematic review. J Infect 2008; 56(5): 381-8.

[http://dx.doi.org/10.1016/j.jinf.2008.02.013] [PMID: 18405976]

[6] Vijayalakshmi AM, Ganesh VRR. Hemophagocytic syndrome associated with dengue hemorrhagic fever. Indian Pediatr 2009; 46(6): 545.

[PMID: 19556676]
[7] Castillo L, Carcillo J. Secondary hemophagocytic lymphohistiocytosis and severe sepsis/ systemic inflammatory response syndrome/ multiorgan dysfunction syndrome/macrophage activation syndrome share common intermediate phenotypes on a spectrum of inflammation. Pediatr Crit Care Med 2009; 10(3): 387-92. [http://dx.doi.org/10.1097/PCC.0b013e3181a1ae08] [PMID: 19325 510]

[8] Rivière S, Galicier L, Coppo $\mathrm{P}$, et al. Reactive hemophagocytic syndrome in adults: A retrospective analysis of 162 patients. Am J Med 2014; 127(11): 1118-25.

[http://dx.doi.org/10.1016/j.amjmed.2014.04.034] [PMID: 24835040]

[9] Ray S, Kundu S, Saha M, Chakrabarti P. Hemophagocytic syndrome in classic dengue Fever. J Glob Infect Dis 2011; 3(4): 399-401. [http://dx.doi.org/10.4103/0974-777X.91068] [PMID: 22224008]

[10] Jordan MB, Allen CE, Weitzman S, Filipovich AH, McClain KL. How I treat hemophagocytic lymphohistiocytosis. Blood 2011; 118(15): 4041-52.

[http://dx.doi.org/10.1182/blood-2011-03-278127] [PMID: 21828139]

[11] Srichaikul T, Punyagupta S, Kanchanapoom T, Chanokovat C, Likittanasombat K, Leelasiri A. Hemophagocytic syndrome in Dengue hemorrhagic fever with severe multiorgan complications. J Med Assoc Thai 2008; 91(1): 104-9.

[PMID: 18386553]

(C) 2019 Thangavelu et al.

This is an open access article distributed under the terms of the Creative Commons Attribution 4.0 International Public License (CC-BY 4.0), a copy of which is available at: https://creativecommons.org/licenses/by/4.0/legalcode. This license permits unrestricted use, distribution, and reproduction in any medium, provided the original author and source are credited. 